\title{
Optimality of the range for which equivalence between certain measures of smoothness holds
}

\author{
by
}

\section{Z. DitZian (Edmonton)}

\begin{abstract}
Recently it was proved for $1<p<\infty$ that $\omega^{m}(f, t)_{p}$, a modulus of smoothness on the unit sphere, and $\widetilde{K}_{m}\left(f, t^{m}\right)_{p}$, a $K$-functional involving the LaplaceBeltrami operator, are equivalent. It will be shown that the range $1<p<\infty$ is optimal; that is, the equivalence $\omega^{m}(f, t)_{p} \approx \widetilde{K}_{m}\left(f, t^{r}\right)_{p}$ does not hold either for $p=\infty$ or for $p=1$.
\end{abstract}

1. Introduction and notations. The moduli of smoothness $\omega^{m}(f, t)_{p}$ (see $[\mathrm{Di}, 99]$ ) are given by

$$
\omega^{m}(f, t)_{L_{p}\left(S^{d-1}\right)}=\omega^{m}(f, t)_{p}=\sup _{\rho \in O_{t}}\left\|\Delta_{\rho}^{m} f\right\|_{L_{p}\left(S^{d-1}\right)}
$$

where $S^{d-1}=\left\{\boldsymbol{x}=\left(x_{1}, \ldots, x_{d}\right): x_{1}^{2}+\cdots+x_{d}^{2}=1\right\}, O_{t}=\{\rho \in S O(d)$ : $\rho \boldsymbol{x} \cdot \boldsymbol{x} \geq \cos t$ for all $\left.\boldsymbol{x} \in S^{d-1}\right\}, S O(d)$ is the group of orthogonal matrices whose determinant equals $1, \Delta_{\rho} f(\boldsymbol{x}) \equiv f(\rho \boldsymbol{x})-f(\boldsymbol{x})$ and $\Delta_{\rho}^{m} f(\boldsymbol{x}) \equiv$ $\Delta_{\rho}\left(\Delta_{\rho}^{m-1} f(\boldsymbol{x})\right)$.

The $K$-functional $\widetilde{K}_{m}\left(f, t^{m}\right)_{p}$ is given by

$$
\begin{aligned}
\widetilde{K}_{m}\left(f, t^{m}\right)_{p} & =\widetilde{K}_{m}\left(f, t^{m}\right)_{L_{p}\left(S^{d-1}\right)} \\
& =\inf \left(\|f-g\|_{L_{p}\left(S^{d-1}\right)}+t^{m}\left\|(-\widetilde{\Delta})^{m / 2} g\right\|_{L_{p}\left(S^{d-1}\right)}\right)
\end{aligned}
$$

where the infimum is taken on all $g$ such that $(-\widetilde{\Delta})^{m / 2} g \in L_{p}\left(S^{d-1}\right)$, and $\widetilde{\Delta}$ is the Laplace-Beltrami operator given by

$$
\begin{array}{ll}
\widetilde{\Delta} f(\boldsymbol{x})=\Delta F(\boldsymbol{x}), \quad \boldsymbol{x} \in S^{d-1}, \\
F(\boldsymbol{x})=f\left(\frac{\boldsymbol{x}}{|\boldsymbol{x}|}\right), \quad \Delta=\frac{\partial^{2}}{\partial x_{1}^{2}}+\cdots+\frac{\partial^{2}}{\partial x_{d}^{2}} .
\end{array}
$$

We recall that

$$
H_{k}=\left\{\varphi_{k}: \widetilde{\Delta} \varphi_{k}=-k(k+d-2) \varphi_{k}\right\}, \quad k=0,1, \ldots,
$$

2010 Mathematics Subject Classification: 42B35, 41A17, 41A63.

Key words and phrases: moduli of smoothness, $K$-functionals, Laplace-Beltrami operator. 
$P_{k} f$ is the projection of $f$ on $H_{k}$ (in the $L_{2}\left(S^{d-1}\right)$ sense) and

$$
(-\widetilde{\Delta})^{\alpha} f=\sum_{k=1}^{\infty}(k(k+d-2))^{\alpha / 2} P_{k} f \quad \text { for } \alpha \neq 0, \alpha \in \mathbb{R} .
$$

It was proved in [Da-Di-Hu] (and for even $m$ in $[\mathrm{Di}, 07]$ ) that $\omega^{m}(f, t)_{p} \approx$ $\widetilde{K}_{m}\left(f, t^{m}\right)_{p}$ for $1<p<\infty$; that is,

$$
C^{-1} \widetilde{K}_{m}\left(f, t^{m}\right)_{p} \leq \omega^{m}(f, t)_{p} \leq C \widetilde{K}_{m}\left(f, t^{m}\right)_{p}, \quad 1<p<\infty .
$$

Here we show that the second inequality of $(1.6)$ does not hold for $p=\infty$ or $p=1$. The first inequality of 1.6 was proved for even $m$ and $1 \leq p \leq \infty$ in [Da-Di-Hu, Th. 9.1] (and for even $d$ and $m$ and many other spaces in Da-Di]).

The main result of this paper is summarized by the next theorem.

Theorem 1.1. The inequality

$$
\omega^{m}(f, t)_{p} \leq C \widetilde{K}_{m}\left(f, t^{m}\right)_{p}
$$

fails for $p=1$ and $p=\infty$ for any $m=1,2, \ldots$.

This failure means that for any integer $m$ and any constant $C$ there exist $f \in L_{1}\left(S^{d-1}\right)$ (for $p=1$ ) and $f \in L_{\infty}\left(S^{d-1}\right)$ (for $p=\infty$ ) for which the inequality is not valid in the range $0<t \leq t_{0}$.

2. A counterexample for $L_{\infty}$. For $L_{\infty}\left(S^{d-1}\right), d \geq 3$ and $m=2$ we use the function

$$
f\left(x, y, u_{1}, \ldots, u_{d-3}, z\right)= \begin{cases}\left(x^{2}-y^{2}\right) \log \left(x^{2}+y^{2}\right), & x \neq 0, y \neq 0 \\ 0 & \text { otherwise }\end{cases}
$$

which is clearly in $L_{\infty}\left(S^{d-1}\right)$. We recall (see [Er, Chapter XI] and [Vi, Ch. IX, p. 494]) that

$$
\begin{aligned}
& r^{-2} \widetilde{\Delta} f=\Delta f-r^{-d+1} \frac{\partial}{\partial r}\left(r^{d-1} \frac{\partial f}{\partial r}\right), \\
& r=\left(x^{2}+y^{2}+u_{1}^{2}+\cdots+u_{d-3}^{2}+z^{2}\right)^{1 / 2},
\end{aligned}
$$

where $\Delta$ is the Laplacian. Straightforward calculation yields

$$
\begin{aligned}
\Delta f & =\frac{10\left(x^{2}-y^{2}\right)}{x^{2}+y^{2}}-\frac{4\left(x^{4}-y^{4}\right)}{\left(x^{2}+y^{2}\right)^{2}} \\
& =\frac{6\left(x^{2}-y^{2}\right)}{x^{2}+y^{2}} \quad \text { and } \quad|\Delta f| \leq 6 .
\end{aligned}
$$


We express $f$ in polar coordinates given by (see [Er, Ch. XI] and [Vi, Ch. IX, p. 435])

$$
\begin{aligned}
z & =r \cos \theta_{1}, \\
u_{d-3} & =r \sin \theta_{1} \cos \theta_{2}, \\
& \vdots \\
u_{1} & =r \sin \theta_{1} \cdots \sin \theta_{d-3} \cos \theta_{d-2}, \\
x & =r \sin \theta_{1} \cdots \sin \theta_{d-2} \cos \varphi, \\
y & =r \sin \theta_{1} \cdots \sin \theta_{d-2} \sin \varphi,
\end{aligned}
$$

where $0 \leq \theta_{i} \leq \pi$ for $1 \leq i \leq d-2$ and $0 \leq \varphi \leq 2 \pi$. (Clearly, for $d=3$, $u_{1}, \ldots, u_{d-3}$ do not exist.) Hence $f\left(r, \theta_{1}, \ldots, \theta_{d-2}, \varphi\right)=r^{2} \cos 2 \varphi \sin ^{2} \theta_{1} \cdots \sin ^{2} \theta_{d-2} \log r^{2} \sin ^{2} \theta_{1} \cdots \sin ^{2} \theta_{d-2}$.

Straightforward computation implies that (for $r=1$ )

$$
\left|r^{-d+1} \frac{\partial}{\partial r}\left(r^{d-1} \frac{\partial f}{\partial r}\right)\right|
$$

is smaller than

$$
C\left(1+\sin ^{2} \theta_{1} \ldots \sin ^{2} \theta_{d-2}\left|\log \left(\sin ^{2} \theta_{1} \cdots \sin ^{2} \theta_{d-2}\right)\right|\right),
$$

which is bounded for all $\theta_{i}$. The above, together with 2.2), implies that $\widetilde{\Delta} f$ is bounded on $S^{d-1}$ (when $r=1$ ) and hence

$$
\widetilde{K}_{2}\left(f, t^{2}\right)_{\infty} \leq C_{1} t^{2} \quad \text { for } f \text { of } 2.1 \text {. }
$$

We will now show that $f$ given in (2.1) satisfies

$$
\omega^{2}(f, t)_{\infty} \geq C_{2} t^{2}|\log t|
$$

Choosing the point $\boldsymbol{\zeta}=(x, y, \ldots, z)=(0, \ldots, 0,-1)$ and the transformation (rotation)

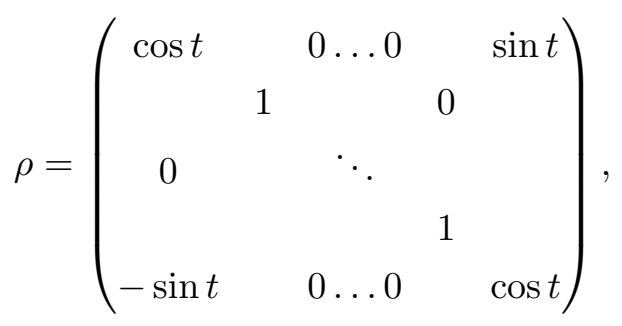

we have

$$
f(\rho \zeta)-2 f(\boldsymbol{\zeta})+f\left(\rho^{-1} \boldsymbol{\zeta}\right)=2 \sin ^{2} t \log \sin ^{2} t,
$$

which establishes (2.5). Therefore, for $L_{\infty}\left(S^{d-1}\right), d \geq 3$ and $m=2$ the right hand inequality of (1.6) is not valid. 
To show that the right hand inequality of 1.6 fails for $m=1$ we assume that it does not fail and hence, for $f \in C^{2}$ and $\rho \in O_{t}$,

$$
\left|\Delta_{\rho} f\right| \leq C t\left\|(-\widetilde{\Delta})^{1 / 2} f\right\|_{L_{\infty}\left(S^{d-1}\right)} .
$$

Iterating the above will cause a contradiction with (2.5). We note that, for $f \in C^{2}\left(S^{d-1}\right)$,

$$
\widetilde{K}_{1}(f, t)_{\infty} \leq C t\left\|(-\widetilde{\Delta})^{1 / 2} f\right\|_{\infty} \quad \text { and } \quad \widetilde{K}_{2}\left(f, t^{2}\right)_{\infty} \leq C t^{2}\|(-\widetilde{\Delta}) f\|_{\infty} .
$$

To our knowledge the case $m=2$ does not imply the failure of the right hand inequality of (1.6) for all $m$. For even $m$, we set $m=2 \ell$ and use the function

$$
f_{2 \ell}\left(x, y, u_{1}, \ldots, u_{d-3}, z\right)= \begin{cases}P_{\ell}(x, y) \log \left(x^{2}+y^{2}\right), & x \neq 0, y \neq 0 \\ 0, & \text { otherwise }\end{cases}
$$

with

$$
P_{\ell}(x, y)=\sum_{k=0}^{\ell} a_{k} x^{2(\ell-k)} y^{2 k}, \quad P_{\ell}(\cos \varphi, \sin \varphi)=\cos 2 \ell \varphi,
$$

where the coefficients $a_{k}$ are determined by $P_{\ell}(\cos \phi, \sin \phi)=\cos 2 \ell \phi$. In Section 4 we show that using the Taylor formula, we will obtain

$$
\omega^{2 \ell}\left(f_{2 \ell}, t\right)_{\infty} \geq C_{2 \ell} t^{2 \ell}|\log t| \quad \text { for } 0<t<t_{0},
$$

and using iteration of $(2.2)$ and some delicate computation, we will obtain

$$
\widetilde{K}\left(f_{2 \ell}, t^{2 \ell}\right)_{\infty} \leq C_{2 \ell}^{*} t^{2 \ell} .
$$

Combining the inequalities 2.9) and 2.10 implies

$$
\omega^{2 \ell}\left(f_{2 \ell}, t\right)_{\infty} \geq A_{2 \ell} \widetilde{K}_{2 \ell}\left(f_{2 \ell}, t^{2 \ell}\right)_{\infty}|\log t| \quad \text { for } 0<t<t_{0} .
$$

For odd $m$ we use 2.9 and 2.10 with $\ell=m$ and follow exactly the considerations for $m=1$.

We note that for $L_{\infty}\left(\mathbb{R}^{d}\right)$ (or $L_{\infty}\left(T^{d}\right)$ ) one has

$$
C^{-1} K_{m}\left(f, t^{m}\right)_{p} \leq \omega^{m}(f, t)_{p} \leq C K_{m}\left(f, t^{m}\right)_{p}, \quad 1<p<\infty,
$$

where translations in $\mathbb{R}^{d}$ (not elements of $S O(d)$ ) are used in the definition of $\omega^{m}(f, t)_{p}$, and the Laplacian (instead of the Laplace-Beltrami operator) is used in the definition of $K_{m}\left(f, t^{m}\right)_{p}$. For $d \geq 2$ and $p=\infty$ the right hand inequality of (2.12) fails because of the failure of the estimate of the Riesz transform (see [St]). The example given in $(2.1)$ or $(2.7)$ can be modified by

$$
\begin{aligned}
& f_{2 \ell}^{*}\left(x, y, u_{1}, \ldots, u_{d-3}, z\right) \\
& \quad=f_{2 \ell}\left(x, y, u_{1}, \ldots, u_{d-3}, z\right) \psi\left(x^{2}+y^{2}+u_{1}^{2}+\cdots+u_{d-3}^{2}+z^{2}\right)
\end{aligned}
$$

where

$$
\psi\left(r^{2}\right)= \begin{cases}1, & \left|r^{2}\right| \leq 1 \\ 0, & \left|r^{2}\right| \geq 2\end{cases}
$$


$\psi \in C^{\infty}$ and $r^{2}=x^{2}+y^{2}+\cdots+z^{2}$. The function $f_{2 \ell}^{*}$ will provide an example for the failure of 2.12 for $d \geq 2, p=\infty$ and $m=2 \ell$ (when $d=2, z$ is eliminated). Following previous arguments, a contradiction can establish the above contention (on the failure of (2.12) for odd $m$ and $p=\infty$.

3. The failure of the inequality for $L_{1}$. For $L_{1}\left(S^{d-1}\right), d \geq 3$, we prove the failure of the right hand inequality of (1.6) by contradiction. We assume $\omega^{m}(H, t)_{1} \leq C \widetilde{K}_{m}\left(H, t^{m}\right)_{1}$ for all $H \in L_{1}\left(S^{d-1}\right)$. Setting $H=$ $(-\widetilde{\Delta})^{-m / 2} g$ for $g \in L_{1}\left(S^{d-1}\right)$ satisfying $P_{0} g=0$ (i.e. $\int_{S^{d-1}} g(x) d x=0$ ), one has $\left\|\Delta_{\rho}^{m}\left\{(-\widetilde{\Delta})^{-m / 2} g\right\}\right\|_{L_{1}\left(S^{d-1}\right)} \leq C t^{m}\|g\|_{L_{1}\left(S^{d-1}\right)}$ for all $\rho \in O_{t}$ where $(-\widetilde{\Delta})^{-m / 2} f$ is given by $(1.5)$. We note that $\Delta_{\rho}$ is not a multiplier operator but that it still commutes with powers of $-\widetilde{\Delta}$, i.e. with $(-\widetilde{\Delta})^{\alpha}(\alpha \in \mathbb{R})$. As established in the last section, for any $M>0$ we have a function $f \in$ $L_{\infty}\left(S^{d-1}\right)$ (and in fact $\left.f \in C^{m}\left(S^{d-1}\right)\right), t>0$ and $\rho \in O_{t}$ such that

$$
\left\|\Delta_{\rho}^{m} f\right\|_{L_{\infty}\left(S^{d-1}\right)} \geq M t^{m}\left\|(-\widetilde{\Delta})^{m / 2} f\right\|_{L_{\infty}\left(S^{d-1}\right)}
$$

and hence for $F=(-\widetilde{\Delta})^{m / 2} f$ (for which $P_{0} F=0$ ),

$$
\left\|\Delta_{\rho}^{m}(-\widetilde{\Delta})^{-m / 2} F\right\|_{L_{\infty}\left(S^{d-1}\right)} \geq M t^{m}\|F\|_{L_{\infty}\left(S^{d-1}\right)} .
$$

We may now choose $G \in L_{1}\left(S^{d-1}\right)$ with $\|G\|_{L_{1}\left(S^{d-1}\right)}=1$ so that

$$
\left\langle G, \Delta_{\rho}^{m}(-\widetilde{\Delta})^{-m / 2} F\right\rangle \geq\left\|\Delta_{\rho}^{m}(-\widetilde{\Delta})^{-m / 2} F\right\|_{L_{\infty}\left(S^{d-}\right)}-\varepsilon
$$

where $\langle\varphi, \psi\rangle=\int_{S^{d-1}} \varphi(x) \psi(x) d x$.

For $g=G-P_{0} G$ which satisfies $\|g\|_{L_{1}\left(S^{d-1}\right)} \leq 2$ and $P_{0} g=0$ we have

$$
\left\langle\Delta_{\rho^{-1}}^{m}\left\{(-\widetilde{\Delta})^{m / 2} g\right\}, F\right\rangle \leq C t^{m}\|g\|_{L_{1}\left(S^{d-1}\right)}\|F\|_{L_{\infty}\left(S^{d-1}\right)} \leq 2 C t^{m}\|F\|_{L_{\infty}\left(S^{d-1}\right)}
$$

as $\rho^{-1} \in O_{t}$ if $\rho \in O_{t}$. However,

$$
\begin{aligned}
\left\langle\Delta_{\rho^{-1}}^{m}\left\{(-\widetilde{\Delta})^{-m / 2} g\right\}, F\right\rangle & =\left\langle g, \Delta_{\rho}^{m}\left\{(-\widetilde{\Delta})^{-m / 2} F\right\}\right\rangle=\left\langle G, \Delta_{\rho}^{m}\left\{(-\widetilde{\Delta})^{-m / 2} F\right\}\right\rangle \\
& \geq\left\|\Delta_{\rho}^{m}\left\{(-\widetilde{\Delta})^{-m / 2} F\right\}\right\|_{L_{\infty}\left(S^{d-1}\right)}-\varepsilon \\
& \geq M t^{m}\|F\|_{L_{\infty}\left(S^{d-1}\right)}-\varepsilon,
\end{aligned}
$$

and this causes a contradiction for $M>3 C$.

For $L_{1}\left(\mathbb{R}^{d}\right)$ or $L_{1}\left(T^{d}\right)(d \geq 2)$ the same argument for the corresponding failure of 2.12 follows and in fact in this case both $\Delta_{h}^{m} f$ and $(-\Delta)^{-m / 2} f$ are multiplier operators which naturally commute.

4. Proof of the inequality (2.11) for $\ell \geq 2$. Using the description of $f_{2 \ell}$ in polar coordinates, i.e.

$$
f_{2 \ell}=r^{2 \ell} \cos 2 \ell \varphi \sin ^{2 \ell} \theta_{1} \cdots \sin ^{2 \ell} \theta_{d-1} \log r^{2} \sin ^{2} \theta_{1} \cdots \sin ^{2} \theta_{d-2},
$$


we have

$$
\begin{aligned}
& r^{2} r^{-d+1} \frac{\partial}{\partial r}\left(r^{d-1} \frac{\partial}{\partial r} f_{2 \ell}\right) \\
& \quad=2 \ell(2 \ell+d-2) f_{2 \ell}+[(2 \ell+d-2)+2 \ell] r^{2 \ell} \cos 2 \varphi \ell \sin ^{2 \ell} \theta_{1} \cdots \sin ^{2 \ell} \theta_{d-2} .
\end{aligned}
$$

To compute $\widetilde{\Delta}$ we also calculate $\Delta f_{2 \ell}$ :

$$
\begin{aligned}
\Delta f_{2 \ell}= & \left(\left(\frac{\partial^{2}}{\partial x^{2}}+\frac{\partial^{2}}{\partial y^{2}}\right) P_{\ell}(x, y)\right) \log \left(x^{2}+y^{2}\right)+2 \frac{\partial}{\partial x} P_{\ell}(x, y) \frac{2 x}{x^{2}+y^{2}} \\
& +2 \frac{\partial}{\partial y} P_{\ell}(x, y) \frac{2 y}{x^{2}+y^{2}}+P_{\ell}(x, y) \frac{8}{x^{2}+y^{2}} .
\end{aligned}
$$

We now observe that

$$
\left(\frac{\partial^{2}}{\partial x^{2}}+\frac{\partial^{2}}{\partial y^{2}}\right) P_{\ell}(x, y)=0 .
$$

This is shown using the two-dimensional description, i.e. $x=\rho \cos \psi, y=$ $\rho \sin \psi$,

$$
P_{\ell}(x, y)=\rho^{2 \ell} \cos 2 \ell \psi \quad \text { and } \quad \frac{\partial^{2}}{\partial x^{2}}+\frac{\partial^{2}}{\partial y^{2}}=\frac{\partial^{2}}{\partial \rho^{2}}+\rho^{-1} \frac{\partial}{\partial \rho}+\rho^{2} \frac{\partial^{2}}{\partial \psi^{2}},
$$

which imply

$$
\begin{aligned}
& \left(\left(\frac{\partial}{\partial x}\right)^{2}+\left(\frac{\partial}{\partial y}\right)^{2}\right) P_{\ell}(x, y) \\
& \quad=2 \ell(2 \ell-1) \rho^{2 \ell-2} \cos 2 \ell \psi+2 \ell \rho^{2 \ell-2} \cos 2 \ell \psi-(2 \ell)^{2} \rho^{2 \ell-2} \cos 2 \ell \psi
\end{aligned}
$$

As $x^{2}+y^{2}=r^{2} \sin ^{2} \theta_{1} \cdots \sin ^{2} \theta_{d-2}$ and $P_{\ell}(x, y)$ is a homogeneous polynomial of degree $2 \ell$ in $x$ and $y$, we can write

$$
\begin{aligned}
r^{2} \Delta f_{2 \ell} & =r^{2 \ell} Q_{\ell}\left(\cos \psi, \sin \varphi, \sin \theta_{1} \sin \theta_{2} \cdots \sin \theta_{d-2}\right) \\
& =r^{2 \ell} Q_{\ell}^{*}(\cos \varphi, \sin \varphi)\left(\sin \theta_{1} \cdots \sin \theta_{d-2}\right)^{2 \ell-2}
\end{aligned}
$$

where $Q_{\ell}^{*}$ is a polynomial in $\cos \varphi$ and $\sin \varphi$.

Therefore, $\widetilde{\Delta}^{\ell-1} r^{2 \ell} Q_{\ell}\left(\cos \varphi, \sin \varphi, \sin \theta_{1} \cdots \sin \theta_{d}\right)$ is bounded using the description of $\widetilde{\Delta}$ in polar coordinates as given in [ $\mathrm{Er}, \mathrm{Ch}$. XI] (see also [Da-Di-Hu, (2.6)] and [Vi, (6), p. 494]). Similarly, $\widetilde{\Delta}^{\ell-1} r^{2 \ell} \cos 2 \ell \varphi \sin ^{2 \ell} \theta_{1} \ldots$ $\sin ^{2 \ell} \theta_{d-2}$ is also bounded. To examine $2 \ell(2 \ell+d-1) \widetilde{\Delta}^{\ell-1} f_{2 \ell}$ we follow the above procedure and obtain, after $\ell-1$ iterations, a constant times $f_{2 \ell}$ plus other terms which are bounded. We note that $f_{2 \ell}$ is bounded (when $r=1$ ) and hence $\left\|\widetilde{\Delta}^{\ell} f_{2 \ell}\right\|_{L_{\infty}\left(S^{d-1}\right)} \leq C$, which implies 2.10 . We now use $\rho$ of 2.6 and $\boldsymbol{\zeta}=(0,0, \ldots, 0,-1)$ and note that $\left\|\Delta_{\rho}^{2 \ell} f_{2 \ell}\right\|_{L_{\infty}\left(S^{d-1}\right)} \geq\left|\Delta_{\rho}^{2 \ell} f_{2 \ell}(\rho \zeta)\right|$. Using $a_{0}=1$ (with $a_{j}$ of (2.8), which follows by setting $\varphi=0$ and then 
using the Taylor formula, we have

$$
\begin{aligned}
\mid \Delta_{\rho}^{2 \ell} f_{2 \ell}\left(\rho^{\ell+1} \zeta\right) & =\left|\sum_{j=-\ell}^{\ell}(-1)^{j}\left(\begin{array}{c}
2 \ell \\
\ell+j
\end{array}\right) f_{2 \ell}\left(\rho^{j+1+\ell} \boldsymbol{\zeta}\right)\right| \\
& =\left|\sum_{j=-\ell}^{\ell}(-1)^{j}\left(\begin{array}{c}
2 \ell \\
\ell+j
\end{array}\right)\left(\sin ^{2 \ell}(j+1+\ell) t\right) \log \sin ^{2}(j+1+\ell) t\right| \\
& =C_{1} t^{2 \ell}\left|\left(\frac{\partial}{\partial t}\right)^{2 \ell}\left(\left(\sin ^{2 \ell} t\right) \log \sin ^{2} t\right)_{t=\eta}\right|
\end{aligned}
$$

where $\eta$ is in $[t,(2 \ell+1) t]$. Since $\sin t \log \sin ^{2} t$ is bounded, we have

$$
\left|\left(\frac{\partial}{\partial t}\right)^{2 \ell}\left(\sin ^{2 \ell} t\right) \log \sin ^{2} t\right|_{t=\eta}=(2 \ell) ! \cos ^{2 \ell} \eta \log \sin ^{2} \eta+g(\eta)
$$

where $g(\eta)$ is bounded. Therefore, for small $t, g(\eta)$ is insignificant compared with $\left|\cos ^{2 \ell} \eta \log \sin ^{2} \eta\right|$. This concludes the proof of (2.9), which, together with 2.10, implies 2.11.

\section{References}

[Da-Di] F. Dai and Z. Ditzian, Jackson inequality for Banach spaces on the sphere, Acta Math. Hungar. 118 (2008), 171-195.

[Da-Di-Hu] F. Dai, Z. Ditzian and H. W. Huang, Equivalence of measures of smoothness in $L_{p}\left(S^{d-1}\right), 1<p<\infty$, Studia Math. 196 (2010), 179-205.

[Di,99] Z. Ditzian, A modulus of smoothness on the unit sphere, J. Anal. Math. 79 (1999), 189-200.

[Di,07] - A note on equivalence of moduli of smoothness on the sphere, J. Approx. Theory 147 (2007), 125-128.

[Er] A. Erdélyi et al., Higher Transcendental Functions, Vol. II, Bateman Manuscript Project, McGraw-Hill, 1953.

[St] E. M. Stein, Singular Integrals and Differentiability Properties of Functions, Princeton Univ. Press, 1970.

[Vi] N. Ya. Vilenkin, Special Functions and the Theory of Group Representations, Transl. Math. Monogr. 22, Amer. Math. Soc., 1968; Russian original: Nauka, Moscow, 1965, and 2nd ed., 1991.

\section{Z. Ditzian}

Department of Mathematical and Statistical Sciences

University of Alberta

Edmonton, AB, Canada T6G 2G1

E-mail: zditzian@math.ualberta.ca 